\title{
A Novel Positron Emission Tomography Imaging Protocol Identifies Seizure-Induced Regional Overactivity of P-Glycoprotein at the Blood-Brain Barrier
}

\author{
Jens P. Bankstahl, ${ }^{1 \star}$ Marion Bankstahl, ${ }^{1 \star}$ Claudia Kuntner, ${ }^{2}$ Johann Stanek, ${ }^{3}$ Thomas Wanek, ${ }^{2}$ Martin Meier, ${ }^{4}$ \\ Xiao-Qi Ding, ${ }^{5}$ Markus Müller, ${ }^{3}$ Oliver Langer, ${ }^{2,3}$ and Wolfgang Löscher ${ }^{1}$ \\ ${ }^{1}$ Department of Pharmacology, Toxicology, and Pharmacy, University of Veterinary Medicine, and Center for Systems Neuroscience, 30559 Hannover, \\ Germany, ${ }^{2}$ Health and Environment Department, Molecular Medicine, AIT Austrian Institute of Technology, 2444 Seibersdorf, Austria, ${ }^{3}$ Department of \\ Clinical Pharmacology, Medical University of Vienna, 1090 Vienna, Austria, and ${ }^{4}$ Department of Cardiology and Angiology and ${ }^{5}$ Institute of Diagnostic and \\ Interventional Neuroradiology, Hannover Medical School, 30625 Hannover, Germany
}

Approximately one-third of epilepsy patients are pharmacoresistant. Overexpression of P-glycoprotein and other multidrug transporters at the blood- brain barrier is thought to play an important role in drug-refractory epilepsy. Thus, quantification of regionally different $\mathrm{P}$-glycoprotein activity in the brain in vivo is essential to identify P-glycoprotein overactivity as the relevant mechanism for drug resistance in an individual patient. Using the radiolabeled P-glycoprotein substrate $(R)-\left[{ }^{11} \mathrm{C}\right]$ verapamil and different doses of coadministered tariquidar, which is an inhibitor of P-glycoprotein, we evaluated whether small-animal positron emission tomography can quantify regional changes in transporter function in the rat brain at baseline and $48 \mathrm{~h}$ after a pilocarpine-induced status epilepticus. P-glycoprotein expression was additionally quantified by immunohistochemistry. To reveal putative seizure-induced changes in blood-brain barrier integrity, we performed gadolinium-enhanced magnetic resonance scans on a 7.0 tesla small-animal scanner. Before P-glycoprotein modulation, brain uptake of $(R)$ - $\left[{ }^{11} \mathrm{C}\right]$ verapamil was low in all regions investigated in control and post-status epilepticus rats. After administration of $3 \mathrm{mg} / \mathrm{kg}$ tariquidar, which inhibits P-glycoprotein only partially, we observed increased regional differentiation in brain activity uptake in post-status epilepticus versus control rats, which diminished after maximal P-glycoprotein inhibition. Regional increases in the efflux rate constant $k_{2}$, but not in distribution volume $V_{\mathrm{T}}$ or influx rate constant $K_{1}$, correlated significantly with increases in P-glycoprotein expression measured by immunohistochemistry. This imaging protocol proves to be suitable to detect seizure-induced regional changes in P-glycoprotein activity and is readily applicable to humans, with the aim to detect relevant mechanisms of pharmacoresistance in epilepsy in vivo.

\section{Introduction}

Approximately one-third of patients suffering from epilepsy is pharmacoresistant, i.e., does not respond to an adequate antiepileptic drug therapy (Regesta and Tanganelli, 1999). Among patients with the most frequent epileptic syndrome, i.e., temporal

\footnotetext{
Received Dec. 18, 2010; accepted April 8, 2011
}

Author contributions: J.P.B., M.B., C.K., M.Me., X.-Q.D., M.Mü., O.L., and W.L. designed research;J.P.B., M.B., C.K., J.S., T.W., M.Me., X.-Q.D., and O.L. performed research; J.P.B., M.B., C.K., and 0.L. analyzed data; J.P.B. and M.B. wrote the paper.

This work was supported by funding from the European Community Seventh Framework Programme (FP7/20072013) under Grant Agreement 201380 ("Euripides") and from the Austrian Science Fund project "Transmembrane Transporters in Health and Disease" (SFB F35). We are grateful to Dr. Joan Abbott, Dr. Alexander Hammers, and Dr. Matthias Koepp for critical reading of this manuscript and helpful suggestions. We thank Gloria Stundner (Austrian Institute of Technology), Dr. Thomas Fillip, and Maria Zsebedics (Seibersdorf Laboratories) for their skilful assistance with animal handling and Severin Mairinger, Bernd Dörner, and Florian Bauer as well as the staff of the radiochemistry laboratory (Seibersdorf Laboratories) for their continuous support. Thomas Flanitzer is gratefully acknowledged for help with data analysis and Dr. Rudolf Karch for advice on compartmental modeling issues.

*J.P.B. and M.B. contributed equally to this work.

The authors declare no competing financial interests.

Correspondence should be addressed to Dr. Jens P. Bankstahl, Department of Pharmacology, Toxicology, and Pharmacy, University of Veterinary Medicine Hannover, Buenteweg 17, 30559 Hannover, Germany. E-mail: jens.bankstahl@tiho-hannover.de.

DOI:10.1523/JNEUROSCI.6616-10.2011

Copyright $\odot 2011$ the authors $\quad 0270-6474 / 11 / 318803-09 \$ 15.00 / 0$ lobe epilepsy, up to $70 \%$ are drug resistant (Kwan and Brodie, 2000). Cumulating evidence suggests that regional overactivity of efflux transporters at the blood-brain barrier $(\mathrm{BBB})$ is one important mechanism contributing to the phenomenon of drug resistance by impeding therapeutically effective concentrations of antiepileptic drugs at their sites of action ("transporter hypothesis") (Löscher and Potschka, 2005a). P-glycoprotein (Pgp), which is physiologically located at the luminal membrane of brain capillary endothelial cells, is currently the most widely studied multidrug transporter. Increased expression of Pgp has been found in epileptogenic brain specimens resected from patients with intractable epilepsy (Tishler et al., 1995; Lazarowski et al., 1999; Sisodiya et al., 2002). Moreover, status epilepticus (SE) or frequent spontaneous seizures in rodent models led to increased expression of Pgp, resulting in decreased brain levels of the antiepileptic drug phenytoin (Rizzi et al., 2002; van Vliet et al., 2007b; Bankstahl and Löscher, 2008), which has been characterized as a Pgp substrate (Potschka and Löscher, 2001; Baltes et al., 2007; Luna-Tortós et al., 2008).

Quantification of Pgp overactivity in epilepsy patients by in vivo imaging would be highly useful because altered treatment strategies, e.g., coadministration of a specific Pgp inhibitor or changeover to a nonsubstrate antiepileptic drug, could then be applied. Up to now, 
there is no validated diagnostic method that allows for in vivo measurement of regionally different Pgp expression and function at the BBB. Several studies demonstrated that positron emission tomography (PET) with ${ }^{11} \mathrm{C}$-radiolabeled Pgp substrates, such as $(R)$ $\left[{ }^{11} \mathrm{C}\right]$ verapamil $(\mathrm{VPM})$, is a promising tool for in vivo investigation of Pgp function at the rat, monkey, and human BBB (Langer et al., 2007; Bankstahl et al., 2008; Liow et al., 2009; Seneca et al., 2009). Low brain uptake of high-affinity Pgp substrates such as VPM, however, limits their suitability as PET tracers for mapping regional overexpression in Pgp activity. We tried to overcome this drawback by performing PET scans after partial inhibition of Pgp. This results in sufficient brain activity uptake for PET imaging without complete Pgp blockade (Kuntner et al., 2010). Thus, regionally specific differences in Pgp expression and functionality after SE may become visible. In the present study, we evaluated whether small-animal PET imaging with VPM after partial Pgp inhibition by tariquidar (TQD) can be used to quantify regional changes in transporter function in the injured rat brain.

\section{Materials and Methods}

Animals. Adult female Sprague Dawley rats (Harlan Nederland) were used for all experiments. As in our previous studies on pilocarpineinduced SE in rats, we used female rats, because they are easier to handle after SE and eliminate various drugs considerably more slowly than male rats (Löscher, 2007). The female rats were housed without males to keep them acyclic or asynchronous with respect to their estrous cycle (Kücker et al., 2010). We have shown previously that this avoids effects of estrous cycle on seizure susceptibility or severity (Wahnschaffe and Löscher, 1992). Animal housing facilities were kept at a temperature of $22 \pm 1^{\circ} \mathrm{C}$ and a humidity of $40-70 \%$. Rats had ad libitum access to food and water and were kept under a $12 \mathrm{~h}$ light/dark cycle. Before being used in the experiments, rats were allowed to adapt to the new conditions for at least 1 week. The study was approved by the institutional animal care and use committees, and all study procedures were performed in accordance with the European Communities Council Directive of November 24, 1986 (86/609/EEC). All efforts were made to minimize both the suffering and the number of animals used in this study.

Chemicals and drugs. Unless otherwise stated, all chemicals were of analytical grade and obtained from Sigma-Aldrich Chemie or Merck and used without additional purification. Isoflurane was obtained from Baxter Vertriebs, TQD from Xenova, diazepam (Faustan) from Temmler Pharma, and dimeglumine gadoterate (Gd-DOTA; Dotarem) from Guerbet. Lithium chloride, methyl scopolamine, pilocarpine, Evan's Blue (EB), and chloral hydrate were dissolved in $0.9 \%$ saline. TQD was freshly dissolved on each experimental day in $2.5 \%$ aqueous dextrose solution. VPM was synthesized from $(R)$-norverapamil (ABX Advanced Biochemical Compounds) and $\left[{ }^{11} \mathrm{C}\right]$ methyl triflate as described previously (Brunner et al., 2005).

SE induction. SE was induced by pilocarpine using a fractionated protocol as described in detail previously (Glien et al., 2001). Briefly, the evening before pilocarpine administration, $127 \mathrm{mg} / \mathrm{kg}$ lithium chloride were administered orally. Twelve to $14 \mathrm{~h}$ later, $1 \mathrm{mg} / \mathrm{kg}$ methyl scopolamine was injected intraperitoneally to reduce peripheral adverse effects of pilocarpine. After 30 $\mathrm{min}, 10 \mathrm{mg} / \mathrm{kg}$ pilocarpine was injected intraperitoneally every half hour until onset of generalized SE. Self-sustaining SE was terminated after $90 \mathrm{~min}$ by intraperitoneal administration of diazepam (up to $25 \mathrm{mg} / \mathrm{kg}$ ).

PET experimental procedure. Full details of the PET experimental procedure were described recently (Kuntner et al., 2010). Throughout the whole experimental procedure, animals were kept under isoflurane anesthesia. One hour before the first PET scan, each animal was implanted with microtubes (Kleinfeld) into the femoral artery and vein to allow repeated arterial blood sampling and administration of TQD and VPM, respectively. After surgery, animals were positioned on a $\mu$ PET bed (animal cradle from Bruker BioSpin) that was kept at $38^{\circ} \mathrm{C}$. A stereotactic holder attached to the bed consisting of ear plugs and a tooth bar was used to fixate the animals' head to ensure a reproducible position. A $\mu$ PET Focus 220 scanner (Siemens Medical Solutions) was used, which consisted of 168 detector modules providing a $7.6 \mathrm{~cm}$ axial and $22 \mathrm{~cm}$ transaxial field of view. Reconstructed image resolu-

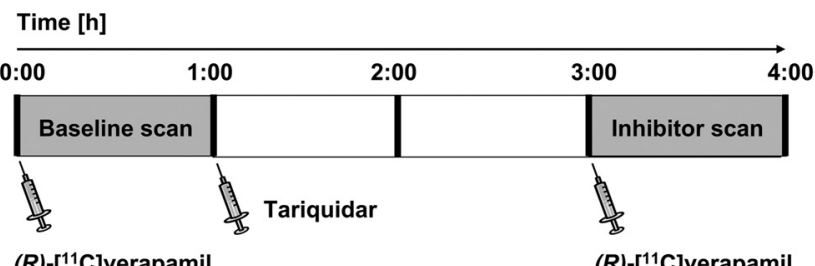

(R)- $\left[{ }^{11} \mathrm{C}\right]$ verapamil

$(R)-\left[{ }^{11} \mathrm{C}\right]$ verapami

Figure 1. Schematic overview of the study setup. After a 60 min baseline PET scan with $(R)$ $\left[{ }^{11} \mathrm{C}\right]$ verapamil, the Pgp inhibitor tariquidar was intravenously administered to control rats or $48 \mathrm{~h}$ post-SE rats. Two hours later, $(R)-\left[{ }^{11} C\right]$ verapamil was injected again and a $60 \mathrm{~min}$ inhibitor scan was acquired.

tion (filtered backprojection) is $1.3 \mathrm{~mm}$ (full-width at half-maximum) in the central field of view and remains under $2 \mathrm{~mm}$ within the central $5-\mathrm{cm}$ diameter field of view. Before each baseline PET scan, a transmission scan using a ${ }^{57}$ Co point source was recorded over $10 \mathrm{~min}$. List mode data were acquired for the defined time period with an energy window of $350-750 \mathrm{keV}$ and 6 ns timing window.

Simultaneously with the first VPM injection, a dynamic 60 min PET scan was started. After this baseline scan, TQD was administered at the halfmaximum effective dose $\left(\mathrm{ED}_{50}\right)$ of $3 \mathrm{mg} / \mathrm{kg}$ (Kuntner et al., 2010) to control rats $(n=6)$ and rats that had exhibited an SE $48 \mathrm{~h}$ before (post-SE rats; $n=$ 6). Additional control $(n=6)$ and post-SE $(n=5)$ rats received $15 \mathrm{mg} / \mathrm{kg}$ TQD for complete Pgp inhibition (Kuntner et al., 2010). Two hours after TQD administration, the second 60 min PET scan (inhibitor scan) was recorded (Fig. 1). During the first $3 \mathrm{~min}$ after radiotracer injection, arterial blood samples were continuously taken using preweighted $2 \mu \mathrm{l}$ micropipettes, followed by additional $10 \mu \mathrm{l}$ samples taken at $5,10,20,30,40$, and 60 min after the beginning of the PET scan. Moreover, one blood sample of 600 $\mu \mathrm{l}$ was collected at $20 \mathrm{~min}$ (baseline scan) and $60 \mathrm{~min}$ (inhibitor scan). Radioactivity in the blood samples was measured in a one-detector Wallac gamma counter (PerkinElmer Life and Analytical Sciences), which was cross-calibrated with the PET camera. The removed blood volume was substituted with approximately the same volume of $0.9 \%$ saline containing 20 $\mathrm{IU} / \mathrm{ml}$ of sodium-heparin. At the end of the inhibitor scan, isoflurane anesthesia was deepened, and rats were killed by drawing a terminal blood sample that was centrifuged for $5 \mathrm{~min}\left(4000 \mathrm{rpm}, 25^{\circ} \mathrm{C}\right.$, Rottanta/TRC centrifuge) to obtain plasma. One aliquot of the plasma sample was directly used to assess metabolism of VPM using a previously described solid-phase extraction assay (Luurtsema et al., 2005; Abrahim et al., 2008). Another aliquot was used to quantify plasma concentrations of TQD as described previously (Wagner et al., 2009).

PET data analysis. PET data from the $60 \mathrm{~min}$ dynamic scans were sorted into three-dimensional sinograms according to the following frame sequence: $8 \times 5 \mathrm{~s}, 2 \times 10 \mathrm{~s}, 2 \times 30 \mathrm{~s}, 3 \times 60 \mathrm{~s}, 2 \times 150 \mathrm{~s}, 2 \times 300 \mathrm{~s}$, and $4 \times 600$ s. PET images were reconstructed by Fourier rebinning of the 3-D sinograms followed by two-dimensional filtered backprojection with a ramp filter, resulting in a voxel size of $0.6 \times 0.6 \times 0.8 \mathrm{~mm}^{3}$. A standard data correction protocol (normalization, attenuation, and decay correction) was applied. A calibration factor for converting units of PET images into absolute radioactivity concentration units was generated by imaging a phantom filled with a known concentration of VPM.

For analysis of PET scans before and after TQD administration, five brain regions of interest (ROIs) (cerebellum, frontal motor cortex, corpus striatum, thalamus, and hippocampus) were manually outlined on multiple coronal MR images of a naive female Sprague Dawley rat using PMOD (pixelwise modeling software; version 2.7.5; PMOD Group) and rigidly coregistered with $\mathrm{PET}$ images as described in detail recently (Kuntner et al., 2010). ROIs were assigned to the PET images and timeactivity curves (TACs), expressed in units of kilobecquerels per milliliters, for the selected brain ROIs were extracted for compartmental modeling. In addition, TACs in individual brain ROIs were normalized to injected radiotracer dose and expressed in units of percentage injected dose per gram tissue $(\% \mathrm{ID} / \mathrm{g})$. From the dose-normalized time activity curves, the area under the curve (AUC) (in $\% \mathrm{ID}^{\star} \mathrm{h} / \mathrm{g}$ ) was calculated using the OriginPro 7.5G software package (OriginLab Corp.). 


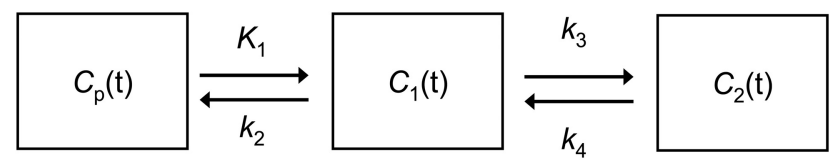

Figure 2. Diagram of the two-tissue four-rate-constant compartmental model used for the kinetic modeling of $(R)-\left[{ }^{11} C\right]$ verapamil PET data. $C_{p}(t)$ is the arterial input function; $C_{1}(t)$ and $C_{2}(t)$ describe the radioactivity concentrations in the first and second tissue compartment. The rate constants $k_{1}, k_{2}, k_{3}$, and $k_{4}$ describe the exchange of radioactivity between the plasma and the first and the second tissue compartments.

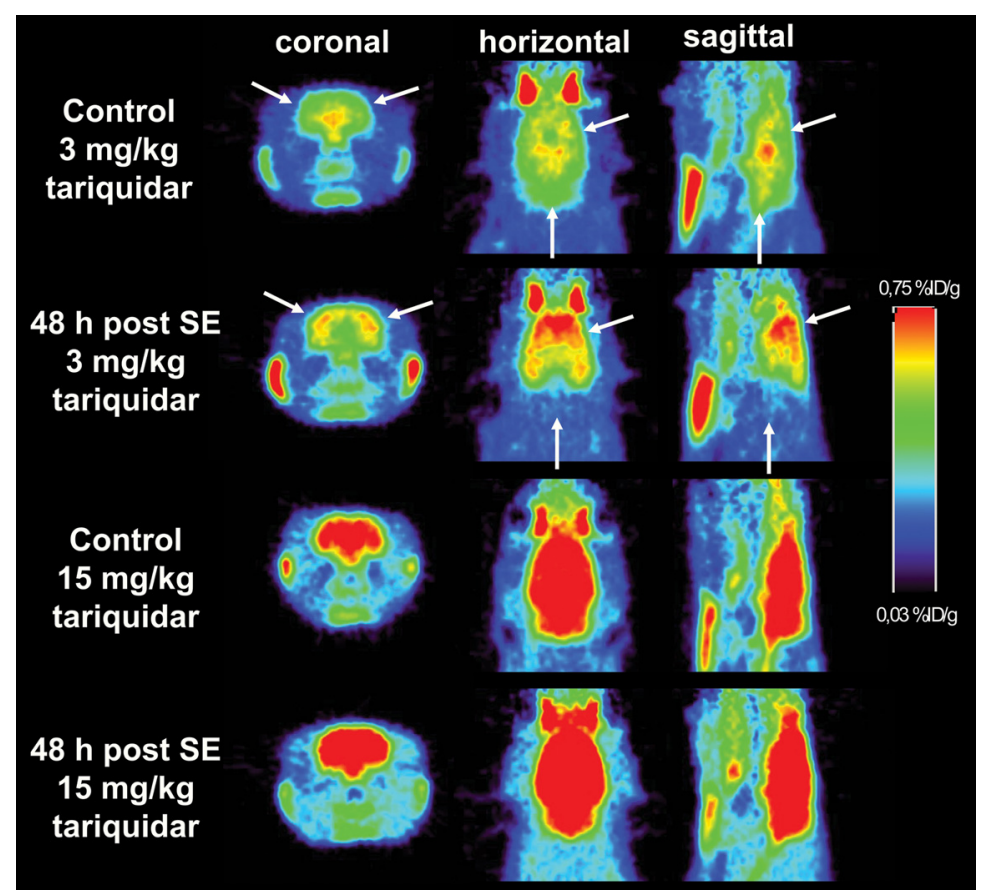

Figure 3. Coronal, horizontal, and sagittal PET summation images $(0-60 \mathrm{~min}$ ) recorded $2 \mathrm{~h}$ after administration of tariquidar ( 3 or 15 $\mathrm{mg} / \mathrm{kg}$ ) in control and post-SE rats. White arrows indicate obvious differences in brain activity uptake in cerebellum and cortical regions.

Kinetic modeling of VPM. Blood activity concentration data were corrected for radioactive decay and converted into plasma concentrations by multiplication with the mean plasma-to-blood activity ratio of all animals at 20 min after tracer injection. Because we found no differences in VPM metabolism between control and post-SE rats (see below), metabolite correction derived from six control rats was applied to activity concentration data at the 10, 20, 30, 40, and 60 min time points (Kuntner et al., 2010). A twotissue four-rate-constant (Fig. 2) compartment model best fitted the VPM time-activity data in rat brain as described previously (Bankstahl et al., 2008). The rate constants $K_{1}$ and $k_{2}$ describe exchange of radioactivity between the plasma and the first tissue compartment, whereas $k_{3}$ and $k_{4}$ describe exchange of radioactivity between the first fast and the second slow tissue compartment (Fig. 2). The used nomenclature is in accordance with the "consensus nomenclature for in vivo imaging" (Innis et al., 2007).

Magnetic resonance imaging. Six additional rats underwent T1- and T2-weighted baseline MR scans before and $48 \mathrm{~h}$ after SE as well as T1weighted scans after administration of Gd-DOTA ( $0.5 \mathrm{mmol} / \mathrm{kg}$, i.v. $)$ to investigate whether severe SE-induced BBB leakage is detectable. MR imaging was conducted on a 7.0 tesla animal scanner with a $38-\mathrm{mm}$ volume coil (T10327V3) serving as both transmitter and receiver coil (Pharmascan 70/16; Bruker BioSpin). Images of coronal sections were acquired by using a $\mathrm{T} 2$-weighted multi-slice, multi-echo sequence with a $5500 \mathrm{~ms}$ repetition time and a $35 \mathrm{~ms}$ echo time, as well as a T1-weighted modified driven equilibrium Fourier transform sequence with a $4000 \mathrm{~ms}$ repetition time and a $3.5 \mathrm{~ms}$ echo time. The field of view used was $3.5 \times$ $3.5 \mathrm{~cm}^{2}$ with a $256 \times 256$ matrix. To provide detailed anatomical structure, a thin-slice thickness of $0.8 \mathrm{~mm}$ was used.
Assessment of albumin extravasation. EB is highly bound to albumin and can be visualized by fluorescence microscopy and is therefore used as a marker of albumin uptake into the brain (van Vliet et al., 2007a). To add a more sensitive method for regional assessment of BBB integrity, EB was administered under a short isoflurane anesthesia $(50 \mathrm{mg} / \mathrm{kg}$ in $4 \mathrm{ml} / \mathrm{kg}$, i.v.) in six control rats and nine rats $48 \mathrm{~h}$ after SE. Two hours later, the animals were transcardially perfused under deep chloral hydrate anesthesia with $4 \%$ paraformaldehyde in $0.1 \mathrm{M}$ phosphate buffer, $\mathrm{pH}$ 7.4. Brains were removed, and $40-\mu \mathrm{m}$-thick coronal sections were cut on a freezing microtome. Slices were covered using a DAPI-containing mounting medium (Dianova) to facilitate regional orientation during fluorescence microscopy. Brain regions corresponding to PET ROIs were visually inspected to evaluate potential cellular EB uptake by a blinded experimenter.

Immunohistochemical labeling and computerassisted quantification of Pgp expression. For immunohistochemical analysis of Pgp expression, additional animals (control, $n=5 ; 48 \mathrm{~h}$ after SE, $n=8$ ) were killed for staining of Pgp in braincapillary endothelial cells. We used a previously published protocol to enhance signal-to-noise ratio for quantification of Pgp expression (Bankstahl and Löscher, 2008). Brains were removed immediately, snap frozen in precooled isopentane, and stored at $-20^{\circ} \mathrm{C}$ until sectioning. Coronal brain sections $(14 \mu \mathrm{m})$ at levels of +3.2 , $-2.3,-3.8,-5.8$, and $-9.6 \mathrm{~mm}$ relative to bregma according to Paxinos and Watson (2007) were cut in a cryostat (Microm) and thaw mounted on HistoBond slides (Marienfeld). All sections of one section level were stained simultaneously to reduce staining variability.

Analysis of the Pgp-labeled surface area in DAB-stained sections was performed by a computer-assisted system as described in detail previously (Volk et al., 2004). Depending on the size of each investigated region (cerebellum, frontal motor cortex, corpus striatum, thalamus, and hippocampus, further separated into CA1, $\mathrm{CA} 3$, dentate gyrus granule cell layer, and dentate hilus), 3-10 fields of $38.321 \mu \mathrm{m}^{2}$ were chosen for analysis of Pgp expression. After standardized adjustment of light intensity and definition of a threshold for every region, the area of Pgp-positive labeling relative to the total area of the fields was measured per region and animal. Because of comparison of different brain section levels, results were normalized using the appropriate control group. In analogy to the PET scan analysis, cerebellum, frontal motor cortex, corpus striatum, thalamus, and hippocampal subregions (CA1, CA3, dentate hilus, and dentate gyrus) were chosen as ROIs for analysis. Mean values were calculated for ROIs that were present in multiple section levels. The mean of the hippocampal subregions was taken for the correlation analysis between outcome parameters of kinetic model analysis and Pgp expression.

Statistical analysis. Group differences of all parameters were analyzed by one-way ANOVA followed by two-tailed Student's $t$ test using GraphPad Prism 5.0 software (GraphPad Software). Pearson's correlation coefficients were calculated for analysis of relationship between changes in modeling outcome parameters and Pgp expression. A $p$ value $\leq 0.05$ was considered significant.

\section{Results}

Quantification of regional VPM brain uptake by small-animal PET

Before TQD administration, brain uptake was generally low, ranging from $1.94 \% \mathrm{ID}^{\star} \mathrm{h} / \mathrm{g}$ in the hippocampus to $3.50 \% \mathrm{ID}^{\star} \mathrm{h} / \mathrm{g}$ in the frontal motor cortex. Baseline brain uptake did not differ between groups, except for the cerebellar region of post-SE rats being reduced by $25.3 \%$ compared with controls (see Fig. $4 A$ ). Maximal Pgp inhibition with $15 \mathrm{mg} / \mathrm{kg}$ (Figs. 3, 4C) resulted in 

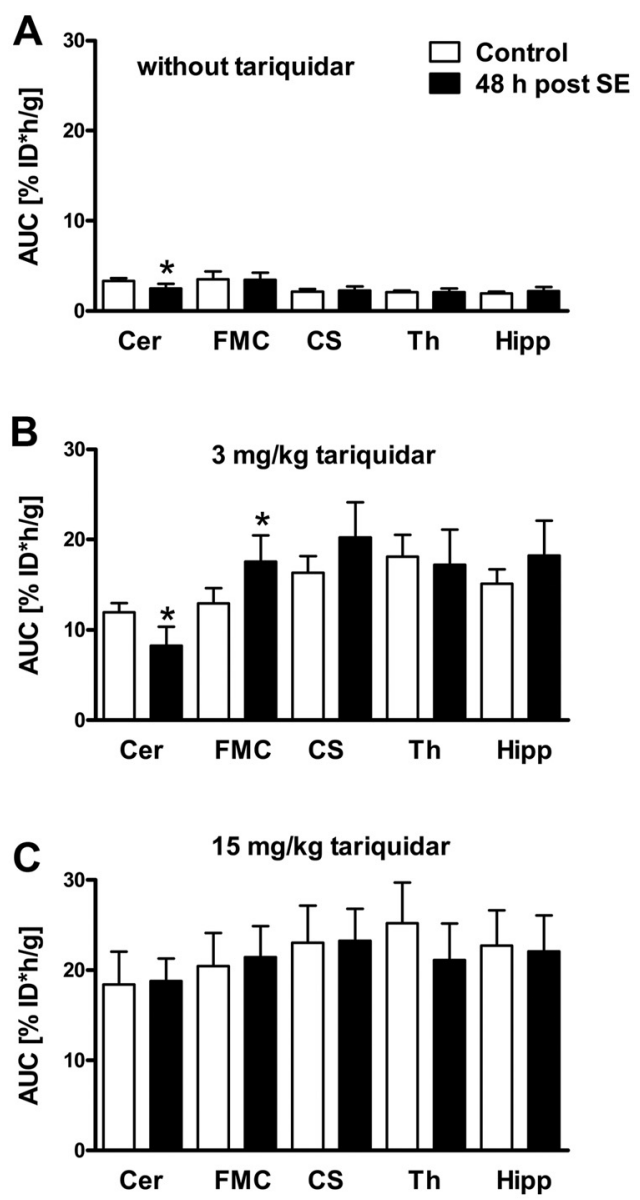

Figure 4. The dose-normalized time-activity AUCs (\% $\left.\% D^{*} \mathrm{~h} / \mathrm{g}, 0-60 \mathrm{~min}\right)$ in control rats and $48 \mathrm{~h}$ post-SE rats in five brain regions during baseline $(\boldsymbol{A}$, without tariquidar) or inhibitor ( $\boldsymbol{B}, 2 \mathrm{~h}$ after administration of $3 \mathrm{mg} / \mathrm{kg}$ tariquidar; $C$, 2 hafter administration of $15 \mathrm{mg} / \mathrm{kg}$ tariquidar) scan. Data are shown as mean $\pm S D$. ${ }^{*} p \leq 0.05$, significant differences between control and post-SE rats. Cer, Cerebellum; FMC, frontal motor cortex; CS, corpus striatum; Th, thalamus; Hipp, hippocampus. $\sim 5.5$-fold increased brain uptake in the cerebellum compared with 12.1-fold increase in the thalamus relative to the baseline scan.

After partial Pgp inhibition with $3 \mathrm{mg} / \mathrm{kg}$ (Figs. 3, 4B), cerebellar brain uptake of post-SE rats was decreased by $30.3 \%$ compared with controls, whereas brain uptake in the frontal motor cortex was increased by $35.9 \%$. Group differences determined with partial Pgp inhibition in the five investigated ROIs disappeared after maximal Pgp inhibition (15 mg/kg) (Figs. 3, 4C). Therefore, this dose level was not further analyzed.

Compartmental modeling of PET data was performed for groups with partial and without Pgp inhibition. Without Pgp modulation by TQD, significant group differences in model outcome parameters were not only observed in the cerebellum but also in the thalamus (Fig. 5, Table 1). A decrease in distribution volume $V_{\mathrm{T}}$ and influx rate constant $K_{1}$ values of 33.2 and $26.8 \%$, respectively, was detected in the cerebellum. In the thalamus, $V_{\mathrm{T}}$ was decreased by $20.4 \%$, whereas the efflux rate constant $k_{2}$ was increased by $39.0 \%$.

After partial Pgp inhibition, group differences became more apparent both in terms of number and extent (Fig. 5, Table 1). We detected $V_{\mathrm{T}}$ decreases in cerebellum $(-41.5 \%)$ and thalamus $(-19.0 \%)$ of post-SE rats. In contrast, we found a $V_{\mathrm{T}}$ increase of $18.6 \%$ in the frontal motor cortex. The influx rate constant $K_{1}$ reflected the $V_{\mathrm{T}}$ group differences only in the cerebellum in terms of a $41.0 \%$ decrease of post-SE rats. The $K_{1}$ values of the four other brain regions were comparable. It is noteworthy that regional $K_{1}$ values after $3 \mathrm{mg} / \mathrm{kg}$ TQD were well below rates of regional cerebral blood flow $(\mathrm{CBF})$ in brain tissue of naive rats under isoflurane anesthesia [e.g., regional cerebral blood flow of $\sim 3.5,4.1$, and $5.5 \mathrm{ml} \cdot \mathrm{g}^{-1} \cdot \mathrm{min}^{-1}$ in hippocampus, cortex, and thalamus, respectively (Hendrich et al., 2001)], indicating that in individual brain regions functional Pgp activity was still present to limit the extraction of VPM. Importantly, partial Pgp inhibition resulted in markedly increased $k_{2}$ values in cerebellum $(+70.7 \%)$, thalamus $(+64.5 \%)$, and hippocampus $(+46.6 \%)$ of post-SE rats compared with control rats. Analysis of $k_{3}$ values did not reveal any group differences between control and post-SE rats neither with nor without Pgp inhibition,
A

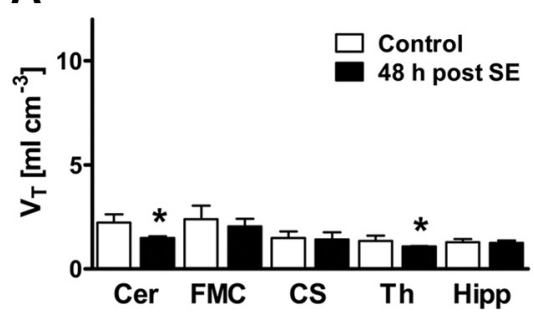

D

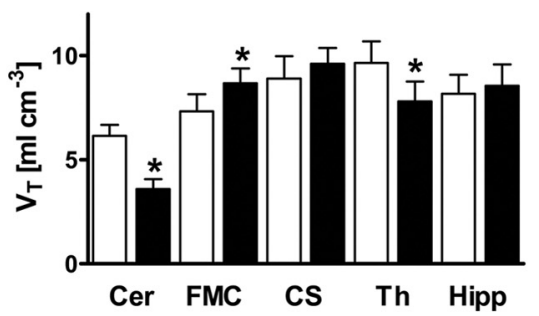

B

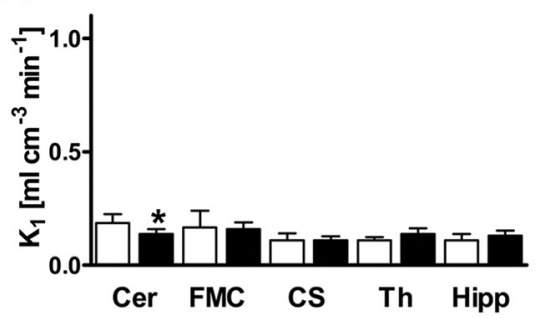

E

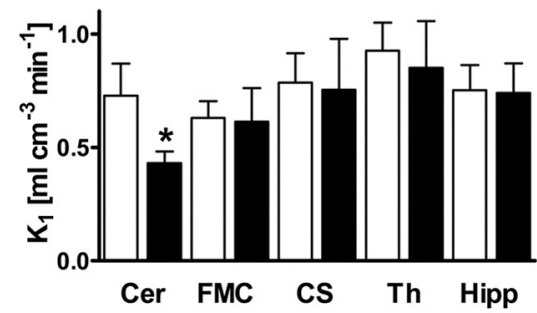

C

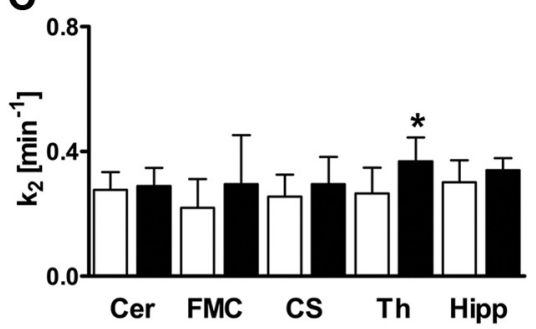

$\mathbf{F}$

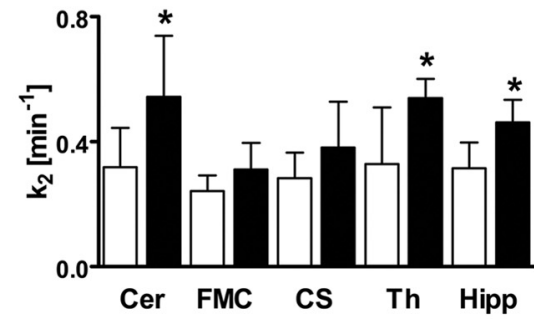

Figure 5. Outcome parameters of kinetic model analysis of PET data for baseline scans (without tariquidar) and inhibitor scans ( $3 \mathrm{mg} / \mathrm{kg}$ tariquidar; $\boldsymbol{A}, \boldsymbol{D}$, distribution volume, $V_{T} ; \boldsymbol{B}, \boldsymbol{E}$, influx rate constant, $K_{1} ; \boldsymbol{C}, \boldsymbol{F}$, efflux rate constant, $k_{2}$ ) of control and post-SE rats in five brain regions. Data are shown as mean \pm SD. ${ }^{*} p \leq 0.05$, Significant differences between control and post-SE rats. Cer, Cerebellum; FMC, frontal motor cortex; CS, corpus striatum; Th, thalamus; Hipp, hippocampus. 
Table 1. $V_{T}, K_{1}, k_{2}, k_{3}$, and $k_{4}$ values derived by compartmental modeling for baseline and inhibitor scans

\begin{tabular}{|c|c|c|c|c|c|c|c|c|}
\hline \multirow[b]{2}{*}{ Brain region } & \multicolumn{4}{|l|}{ Control } & \multicolumn{4}{|l|}{ Post-SE } \\
\hline & $\begin{array}{l}V_{T} \\
\left(\mathrm{ml} \cdot \mathrm{cm}^{-3}\right)\end{array}$ & $\begin{array}{ll}K_{1} & k_{2} \\
\left(\mathrm{ml} \cdot\left(\mathrm{m}^{-3} \mathrm{~min}^{-1}\right)\right. & \left(\mathrm{min}^{-1}\right)\end{array}$ & $\begin{array}{l}k_{3} \\
\left(\min ^{-1}\right)\end{array}$ & $\begin{array}{l}k_{4} \\
\left(\min ^{-1}\right)\end{array}$ & $\begin{array}{l}V_{T} \\
\left(\mathrm{ml} \cdot \mathrm{cm}^{-3}\right)\end{array}$ & $\begin{array}{ll}K_{1} & k_{2} \\
\left(\mathrm{ml} \cdot \mathrm{cm}^{-3} \mathrm{~min}^{-1}\right) & \left(\mathrm{min}^{-1}\right)\end{array}$ & $\begin{array}{l}k_{3} \\
\left(\min ^{-1}\right)\end{array}$ & $\begin{array}{l}k_{4} \\
\left(\min ^{-1}\right)\end{array}$ \\
\hline
\end{tabular}

Baseline scan

$\begin{array}{lllllllll}\text { Cerebellum } & 2.23 \pm 0.40(5.2) & 0.19 \pm 0.04(12.8) & 0.28 \pm 0.06(30.1) & 0.11 \pm 0.05(35.9) & 0.05 \pm 0.02(19.3) & 1.49 \pm 0.09(6.2)^{*} & 0.14 \pm 0.02(13.5)^{*} \quad 0.29 \pm 0.06(30.1) & 0.10 \pm 0.04(37.2) \quad 0.05 \pm 0.02(19.2)\end{array}$

Frontal motor $2.38 \pm 0.67(6.0) \quad 0.17 \pm 0.07(12.3) \quad 0.22 \pm 0.09(33.1) \quad 0.12 \pm 0.07(41.1) \quad 0.05 \pm 0.02(24.3) \quad 2.04 \pm 0.37(6.1) \quad 0.16 \pm 0.03(15.6) \quad 0.29 \pm 0.15(38.8) \quad 0.14 \pm 0.09(44.6) \quad 0.06 \pm 0.02(23.4)$ cortex

$\begin{array}{llllllllll}\text { Corpus striatum } 1.50 \pm 0.31(7.8) & 0.11 \pm 0.03(15.5) & 0.26 \pm 0.07(39.5) & 0.11 \pm 0.05(45.2) & 0.04 \pm 0.02(25.0) & 1.42 \pm 0.35(6.1) & 0.11 \pm 0.02(15.8) & 0.29 \pm 0.09(38.8) & 0.12 \pm 0.07(60.2) & 0.05 \pm 0.02(22.5)\end{array}$

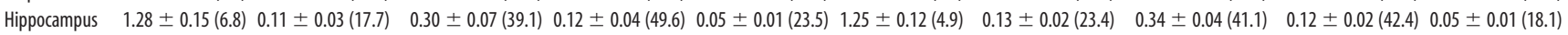

Thalamus $\quad 1.35 \pm 0.25(7.9) \quad 0.11 \pm 0.01(18.7) \quad 0.26 \pm 0.08(36.8) \quad 0.10 \pm 0.04(55.2) \quad 0.05 \pm 0.01(35.2) \quad 1.08 \pm 0.03(4.9)^{*} \quad 0.14 \pm 0.03(23.2)^{*} \quad 0.37 \pm 0.08(43.7) \quad 0.12 \pm 0.06(49.3) \quad 0.03 \pm 0.01(23.6)^{*}$

Inhibitor scan

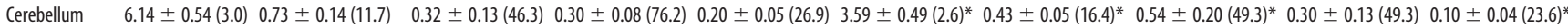

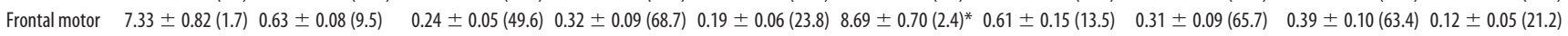

cortex

Corpus striatum $8.89 \pm 1.08(2.7) \quad 0.79 \pm 0.13(10.5) \quad 0.28 \pm 0.08(46.6) \quad 0.47 \pm 0.22(53.4) \quad 0.22 \pm 0.06(23.1) \quad 9.60 \pm 0.77(2.1) \quad 0.76 \pm 0.23(12.1) \quad 0.38 \pm 0.15(53.8) \quad 0.42 \pm 0.08(51.8) \quad 0.12 \pm 0.06(22.0)^{*}$

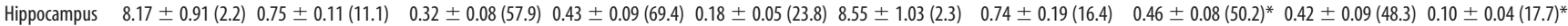

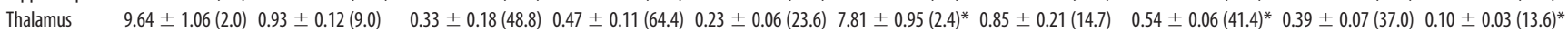

Values are given as mean \pm SD. Significant differences between control and post-SE rats are indicated ( ${ }^{*} p \leq 0.05$ ). The value in parentheses represents the precision of parameter estimates (expressed as their coefficient of variation in percentage), averaged over all rats per group.

A

Control
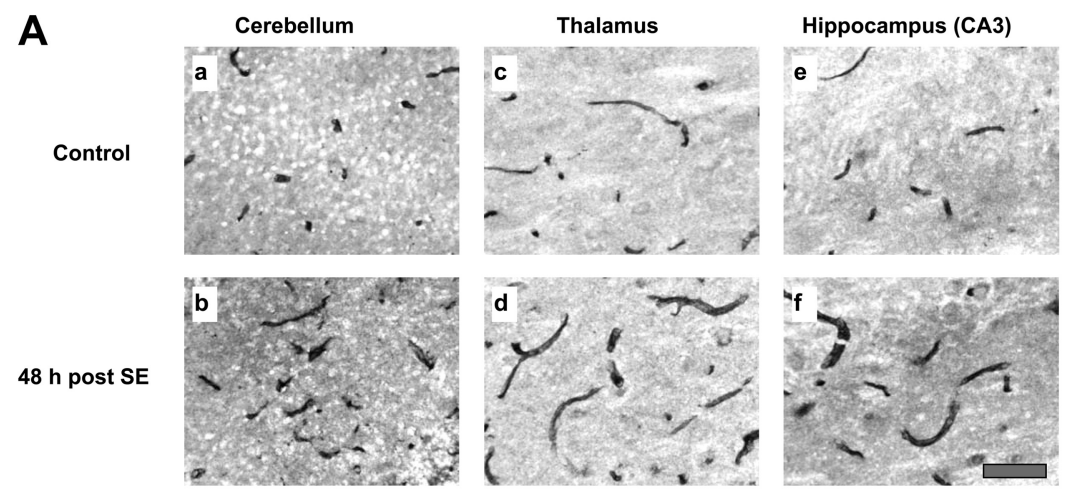

B

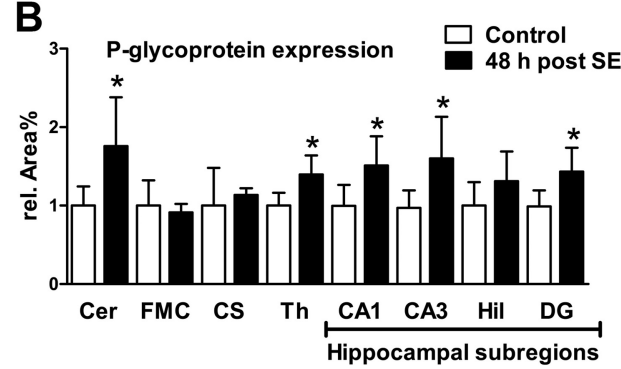

C

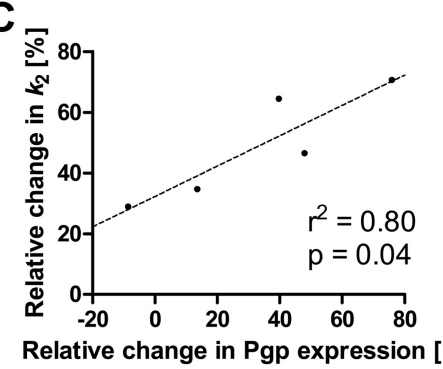

respectively. Plasma levels of TQD at 180 min after administration of the $3 \mathrm{mg} / \mathrm{kg}$ dose were higher in post-SE rats $(0.718 \pm$ $0.074 \mathrm{mg} / \mathrm{ml}$ ) compared with control rats $(0.537 \pm 0.090 \mathrm{mg} / \mathrm{ml})$.

\section{Quantification of Pgp expression by immunohistochemistry}

Pgp was only stained in brain capillary endothelial cells but in neither neurons nor astroglia under the conditions of the immunohistochemical protocol used for this study. Figure $6 \mathrm{~A}$ illustrates representative immunohistochemical Pgp stainings in cerebellar $(a, b)$, thalamic $(c, d)$, and hippocampal $(e, f)$ braincapillary endothelial cells, illustrating a clear SE-induced increase of Pgp-labeled area. The Pgp-labeled area was significantly higher in the cerebellum $(+75.9 \%)$, thalamus $(+39.7 \%)$, CA1 $(+51.5 \%), \mathrm{CA} 3(+64.7 \%)$, and dentate gyrus $(+45.0 \%)$ but remained unchanged in the other investigated regions (Fig. 6B). The increased regional expression of Pgp determined by immunohistochemistry perfectly matched the increased efflux rate constant $k_{2}$ values determined by PET (compare Fig. 5F, Table 2). A correlation analysis between SE-induced increases in Pgp expression and in outcome parameters of kinetic model analysis reached significance only for $k_{2}$ values after partial Pgp inhibition $\left(r^{2}=0.80 ; p=0.04\right)$ (Fig. $6 C$ ).

whereas a global decrease of $k_{4}$ was observed in post-SE rats after partial Pgp inhibition. Nevertheless, partial Pgp inhibition led to globally increased $k_{3}$ values in both control and post-SE rats (Table 1).

VPM metabolism did not differ significantly between control and post-SE rats. At $20 \mathrm{~min}$ after tracer injection during baseline scan, the percentage of unchanged VPM and its lipophilic $\left[{ }^{11} \mathrm{C}\right]$ metabolites was $66.1 \pm 3.9 \%(n=5)$ in control rats and $70.7 \pm 4.0 \%$ $(n=6)$ in post-SE rats. At the end of the inhibitor scan $(3 \mathrm{mg} / \mathrm{kg}$ TQD), these values amounted to $70.5 \pm 13.1$ and $77.0 \pm 6.3 \%$,

\section{MR imaging with Gd-DOTA as contrast agent}

This was performed in six rats before and $48 \mathrm{~h}$ after SE. Figure $7 \mathrm{~A}$ exemplarily shows $\mathrm{T} 1$-weighted MR images of an individual rat in which the choroid plexus and pituitary gland were the only regions with an obviously enhanced signal after Gd-DOTA injection. These observations suggest that there was no major leakage of BBB that may explain the observed group differences in brain activity uptake in the PET experiments after SE. This conclusion is substantiated by 
the fact that, after complete Pgp inhibition by TQD, VPM uptake into the brain did not differ between controls and post-SE rats (Fig. $4 C$ ). However, as shown in Figure 7A, T2-weighted images after SE reveal a striking signal enhancement in hippocampus and piriform cortex as well as clearly reduced CSF space, which was attributable to cell edema, indicating severe pathological changes in the brain after SE.

\section{Assessment of albumin extravasation}

This was performed in six controls and nine rats $48 \mathrm{~h}$ after SE. In part of the SE rats, the visual inspection of brain slices resulted in differentially pronounced red fluorescence in neurons of the dentate hilus, CA1, and CA3, as well as in thalamic subregions but not in cerebellum, frontal motor cortex, or corpus striatum. Striking interindividual differences were found: one rat showed a high amount of fluorescent neurons and circumscribed extracellular fluorescence in hippocampal and thalamic subregions, in five rats intraneuronal fluorescence was found in the same brain regions but in lower extent (Fig. $7 B$ ), and in three SE rats no fluorescence at all was detected. Albumin extravasation did not occur in any of control animals.

\section{Discussion}

Using a novel imaging protocol composed of VPM-PET scans after half-maximal Pgp inhibition by TQD in control rats and rats $48 \mathrm{~h}$ after SE, we were able to quantify group differences in regional Pgp function. This is the first study investigating Pgp activity with VPM-PET in rats after a severe brain insult that can lead to development of epilepsy in animal models as well as in humans. We also demonstrate that changes in outcome parameters of kinetic model analysis correlate with disease-induced changes in Pgp expression.

Our major findings are as follows. First, after partial Pgp inhibition, the extent and number of SE-induced group differences within outcome parameters of kinetic model analysis clearly increased. Second, changes in Pgp function are notably mirrored by both changes in the efflux rate constant $k_{2}$ and Pgp expression levels, which correlate significantly. Third, increased Pgp expression and activity after SE occurs not only in primary epileptogenic regions, such as the hippocampus, but also in the thalamus and the cerebellum.

\section{Seizure-induced overexpression of Pgp}

Because accessibility to brain tissue of epilepsy patients is very limited, animal models are mandatory tools for studying mechanisms of pharmacoresistant epilepsy (Löscher and Potschka, 2005a) and essential for the neurobiological evaluation of imaging protocols. By using Pgp immunohistochemistry, we recently demonstrated an increase in Pgp expression at the BBB of rats $48 \mathrm{~h}$ after SE in hippocampal subregions (Bankstahl and Löscher, 2008), which we extended to brain regions that are often not the focus in basic epilepsy research. Importantly, we and others found decreased brain
Table 2. Summary of statistically significant SE-induced changes in Pgp outcome parameters of kinetic model analysis of PET data (before and after partial Pgp inhibition) and in Pgp expression (as determined by immunohistochemistry)

\begin{tabular}{|c|c|c|c|c|c|c|c|}
\hline \multirow[b]{2}{*}{ Region of interest } & \multicolumn{3}{|c|}{ Baseline scan } & \multicolumn{3}{|c|}{ Inhibitor scan } & \multirow[b]{2}{*}{ Pgp expression } \\
\hline & $V_{T}$ & $K_{1}$ & $k_{2}$ & $V_{T}$ & $K_{1}$ & $k_{2}$ & \\
\hline Cerebellum & $\downarrow$ & $\downarrow$ & NS & $\downarrow$ & $\downarrow$ & $\uparrow$ & $\uparrow$ \\
\hline Frontal motor cortex & NS & NS & NS & $\uparrow$ & NS & NS & NS \\
\hline Corpus striatum & NS & NS & NS & NS & NS & NS & NS \\
\hline Thalamus & $\downarrow$ & NS & $\uparrow$ & $\downarrow$ & NS & $\uparrow$ & $\uparrow$ \\
\hline Hippocampus & NS & NS & NS & NS & NS & $\uparrow$ & $\uparrow$ \\
\hline
\end{tabular}

NS, Not significant.

Figure 7. A, Coronal T2-and T1-weighted MR images before (top) and $48 \mathrm{~h}$ post-SE (bottom). The T2-weighted image post-SE ima shows reduced CSF space, both indicating cell edema. The third column shows T1-weighted scans after a Gd-DOTA bolus inection. Post-Gd images show enhancement in the choroid plexus and pituitary gland (arrows) but no hyperintensity in the xtravasation. In control rats (left), no EB fluorescence was observed microscopically. Uptake of EB in post-SE rats (right) in polymorphic neurons of the dentate hilus is exemplarily shown.

levels of the anticonvulsive Pgp substrate phenytoin in parallel with increased Pgp protein or Mdr1 mRNA levels shortly after SE as well as in chronically epileptic animals (Rizzi et al., 2002; van Vliet et al., 2007b; Bankstahl and Löscher, 2008), further supporting the assumption that the detected overexpression of Pgp has functional consequences. It has been hypothesized that Pgp overexpression could also be induced by prolonged SE in humans (Sisodiya and Thom, 2003; Iannetti et al., 2005; Bankstahl and Löscher, 2008). In apparent line with this possibility, coadministration of verapamil acting as competitive Pgp inhibitor reversed resistance to phenobarbital or phenytoin in two patients with long-lasting refractory SE (Iannetti et al., 2005; Schmitt et al., 2007).

\section{Imaging changes in Pgp function}

A new study design: VPM-PET after half-maximal inhibition of $P g p$

Because Pgp overexpression in the epileptic focus region is considered as one important mechanism of pharmacoresistance in epilepsy patients (Löscher and Potschka, 2005b), a validated 
method for in vivo detection of regionally increased Pgp activity would be a valuable tool for predicting the individual risk of an epilepsy patient not responding to pharmacological treatment with antiepileptic drugs that are Pgp substrates. Although several radiotracers have been used to image cerebral Pgp function (Zoghbi et al., 2008; Syvänen et al., 2009; Kreisl et al., 2010; la Fougère et al., 2010), radiolabeled verapamil is still the most widely studied one (Pike, 2009). However, because VPM is a high-affinity Pgp substrate, its brain uptake is very low, so that small differences in regional Pgp activity cannot easily be measured with this radiotracer (Kuntner et al., 2010). Because complete transporter inhibition would result in VPM brain uptake driven only by passive diffusion, partial Pgp inhibition before tracer administration could be an encouraging paradigm. Therefore, we used the $\mathrm{ED}_{50}$ of the third-generation Pgp inhibitor TQD (Kuntner et al., 2010).

\section{Regional changes in Pgp function}

In line with our hypothesis, no AUC group differences could be detected after complete Pgp inhibition ( $15 \mathrm{mg} / \mathrm{kg}$ TQD), i.e., the efflux transporter was chemically "knocked out," resulting in equally high tracer uptake in all analyzed brain regions (Fig. 4C). Additionally, before Pgp modulation, we observed low brain uptake of radioactivity, demonstrated by low $V_{\mathrm{T}}$ values for both groups of rats (Fig. 5A). The cerebellar decrease in $V_{\mathrm{T}}$ and influx rate constant $K_{1} 48 \mathrm{~h}$ after SE seen in the baseline scans could be interpreted as very high Pgp activity in this brain region. This interpretation is supported by our immunohistological results, in which increase in Pgp expression after SE was highest in the cerebellum (Fig. 6B). Moreover, in scans after half-maximal Pgp inhibition, an even more pronounced cerebellar decrease in $V_{\mathrm{T}}$ and $K_{1}$ and an increase in the efflux rate constant $k_{2}$ could be observed in $48 \mathrm{~h}$ post-SE rats, again pointing to an increased transporter activity in cerebellum (Fig. 5). This was the case despite the fact that the increased TQD levels measured in the post-SE rats would act in the opposite direction.

\section{Reduced influx or increased efflux?}

Increased expression of Pgp at the BBB would be expected to increase the efflux of Pgp substrates from the brain (Löscher and Potschka, 2005a). SE-induced increase in Pgp expression was indeed associated with significant changes of kinetic modeling parameters in affected brain regions. $K_{1}$ and $k_{2}$ describe exchange of radioactivity at the luminal membrane of the $\mathrm{BBB}$. The second, deeper compartment described by $k_{3}$ and $k_{4}$ is seen only after Pgp inhibition (Bankstahl et al., 2008; Wagner et al., 2009), but the distinct physiological correlate remains unclear so far.

Among all calculated PET parameters, $k_{2}$ values after partial Pgp inhibition in post-SE rats correlated best with the immunohistological data (Fig. $6 F$ ). It is still a matter of debate which of the two rate constants for transport of Pgp-substrate tracers across the BBB is most affected by changes in Pgp function.

Two different modes of action for Pgp have been suggested, i.e., influx hindrance (resulting in low $K_{1}$ ) and efflux enhancement (resulting in high $k_{2}$ ). First, a vacuum cleaner model has been suggested (Higgins and Gottesman, 1992), resulting in influx hindrance. In this model, substrates are transported back from the lipid layers of the luminal cell membrane into the blood before they reach the cytoplasm. Second, substrates could be transported from the cytoplasm into the blood. It has also been suggested that Pgp may act by both mechanisms simultaneously (Stein et al., 1994; Sharom, 1997; Higgins and Linton, 2004). Before Pgp inhibition, brain uptake of VPM is very low, which consequently results in low influx and efflux rate constants $\left(K_{1}\right.$, $k_{2}$ ). Probably, most VPM does not reach the cytoplasm and is directly transported back into the blood. However, after partial Pgp inhibition, a considerable amount of VPM enters the first compartment of the brain and is only then sufficiently available for efflux out of the brain by the second mode of action. This could finally lead to an increase in efflux rate $k_{2}$, as observed in this study, despite partial inhibition of Pgp.

In this respect, it is interesting to note that several previous studies in healthy volunteers or rats found that pharmacological inhibition of Pgp caused an increase in the influx rate constant $K_{1}$ but no change in the $k_{2}$ parameter (Liow et al., 2009; Muzi et al., 2009; Wagner et al., 2009; Kreisl et al., 2010; Kuntner et al., 2010). However, Bauer et al. (2009) showed that age-dependent increase in VPM $V_{\mathrm{T}}$ in healthy subjects was mirrored by a decrease in $k_{2}$ but not by an increase in $K_{1}$. In patients suffering from drugresistant unilateral temporal lobe epilepsy, Langer et al. (2007) found increases in VPM $k_{2}$ ipsilateral to the epileptic focus. Hence, for translation of our paradigm to epilepsy patients, both influx and efflux rate constants should be taken into account.

\section{Possible limitations: $C B F$ and integrity of the $B B B$}

On the one hand, the unexpected absence of decreased $V_{\mathrm{T}}$ values in hippocampus could to some extent be attributable to spillover of radioactivity from adjacent structures, such as choroid plexus, which showed high radioactivity uptake as it was also reported for human subjects (Langer et al., 2007). On the other hand, it cannot be ruled out that part of the differences between control and post-SE rats is related to regional changes in CBF after SE. However, given the magnitude of the differences and their correlation with regional Pgp expression levels, it seems unlikely that these changes were only related to CBF. Because regions with higher blood flow would have greater radioactivity uptake, this could at least partially explain why increased hippocampal Pgp levels after SE did not result in decreased $V_{\mathrm{T}}$ of VPM after partial Pgp inhibition in our study. Notably, a recent study by Choy et al. (2010) revealed clear increases of CBF in hippocampus $2 \mathrm{~d}$ after pilocarpine-induced SE. Unfortunately, only one additional brain region corresponding to our ROIs, i.e., thalamus, was investigated in this study, without finding SE-mediated changes in CBF (Choy et al., 2010). Additional studies are needed to reveal possible flow dependency of VPM uptake in additional brain regions.

To clarify whether changes in activity uptake in post-SE rats may be dependent on transient opening of the BBB, rather than on differences in Pgp activity, we performed T1-weighted MRI scans after injection of Gd-DOTA, which is a paramagnetic MRI contrast agent used routinely for evaluation of $\mathrm{BBB}$ lesions in human patients. In our study, T1-weighted MR scans after GdDOTA injection did not reveal major BBB breakdown in any ROI as exemplarily shown in Figure 7A. This is in line with previous MR imaging studies in which no or only circumscribed breakdown of BBB 2-3 h after SE in rodents has been described (Bouilleret et al., 2000; Roch et al., 2002; Hsu et al., 2007; Immonen et al., 2008), which disappeared 6-24 h after SE (Roch et al., 2002; Immonen et al., 2008). Furthermore, low $V_{\mathrm{T}}$ values in VPM baseline scans without marked regional differentiation argue against unselective BBB opening (Fig. 5A).

A recent study detected increased permeability of the rat $\mathrm{BBB}$ after SE and spontaneous recurrent seizures, measured by uptake of fluorescence-labeled albumin into the brain (van Vliet et al., 2007a). In line with the results of this study, we found limited uptake of EB, a marker of albumin uptake, in 
two of the five PET ROIs, i.e., hippocampus and thalamus, and only in $66 \%$ of post-SE rats. Our finding that, after chemical knock-out of Pgp by TQD, VPM uptake is the same in controls and SE rats (Fig. 4C) also argues against any severe impairment of the BBB $48 \mathrm{~h}$ after SE.

\section{Future directions}

In summary, we established a VPM small-animal PET protocol that is suitable to quantify changes in Pgp activity in distinct brain regions after a brain insult that often leads to epilepsy in humans. Two recent pilot studies in healthy volunteers indicate that the PET paradigm described in this paper could be translated to human subjects (Wagner et al., 2009; Bauer et al., 2010). Our protocol is currently used to analyze regional Pgp activity in pharmacoresistant rats and will be expanded to epilepsy patients. Hopefully, it may ultimately serve as a predictive tool for detection of Pgp-mediated pharmacoresistance in epilepsy.

\section{References}

Abrahim A, Luurtsema G, Bauer M, Karch R, Lubberink M, Pataraia E, Joukhadar C, Kletter K, Lammertsma AA, Baumgartner C, Müller M, Langer O (2008) Peripheral metabolism of (R)-[C-11]verapamil in epilepsy patients. Eur J Nucl Med Mol Imaging 35:116-123.

Baltes S, Gastens AM, Fedrowitz M, Potschka H, Kaever V, Löscher W (2007) Differences in the transport of the antiepileptic drugs phenytoin, levetiracetam and carbamazepine by human and mouse P-glycoprotein. Neuropharmacology 52:333-346.

Bankstahl JP, Löscher W (2008) Resistance to antiepileptic drugs and expression of P-glycoprotein in two rat models of status epilepticus. Epilepsy Res 82:70-85.

Bankstahl JP, Kuntner C, Abrahim A, Karch R, Stanek J, Wanek T, Wadsak W, Kletter K, Müller M, Löscher W, Langer O (2008) Tariquidar-induced P-glycoprotein inhibition at the rat blood-brain barrier studied with (R)C-11-verapamil and PET. J Nucl Med 49:1328-1335.

Bauer M, Karch R, Neumann F, Abrahim A, Wagner CC, Kletter K, Müller M, Zeitlinger M, Langer O (2009) Age dependency of cerebral P-gp function measured with (R)-[C-11]verapamil and PET. Eur J Clin Pharmacol 65:941-946.

Bauer M, Karch R, Neumann F, Wagner CC, Kletter K, Müller M, Löscher W, Zeitlinger M, Langer O (2010) Assessment of regional differences in tariquidar-induced P-glycoprotein modulation at the human bloodbrain barrier. J Cereb Blood Flow Metab 30:510-515.

Bouilleret V, Nehlig A, Marescaux C, Namer IJ (2000) Magnetic resonance imaging follow-up of progressive hippocampal changes in a mouse model of mesial temporal lobe epilepsy. Epilepsia 41:642-650.

Brunner M, Langer O, Sunder-Plassmann R, Dobrozemsky G, Müller U, Wadsak W, Krcal A, Karch R, Mannhalter C, Dudczak R, Kletter K, Steiner I, Baumgartner C, Müller M (2005) Influence of functional haplotypes in the drug transporter gene $\mathrm{ABCB} 1$ on central nervous system drug distribution in humans. Clin Pharmacol Ther 78:182-190.

Choy M, Cheung KK, Thomas DL, Gadian DG, Lythgoe MF, Scott RC (2010) Quantitative MRI predicts status epilepticus-induced hippocampal injury in the lithium-pilocarpine rat model. Epilepsy Res 88:221-230.

Glien M, Brandt C, Potschka H, Voigt H, Ebert U, Löscher W (2001) Repeated low-dose treatment of rats with pilocarpine: low mortality but high proportion of rats developing epilepsy. Epilepsy Res 46:111-119.

Hendrich KS, Kochanek PM, Melick JA, Schiding JK, Statler KD, Williams DS, Marion DW, Ho C (2001) Cerebral perfusion during anesthesia with fentanyl, isoflurane, or pentobarbital in normal rats studied by arterial spin-labeled MRI. Magn Reson Med 46:202-206.

Higgins CF, Gottesman MM (1992) Is the multidrug transporter a flippase. Trends Biochem Sci 17:18-21.

Higgins CF, Linton KJ (2004) The ATP switch model for ABC transporters. Nat Struct Mol Biol 11:918-926.

Hsu YH, Lee WT, Chang C (2007) Multiparametric MRI evaluation of kainic acid-induced neuronal activation in rat hippocampus. Brain 130:3124-3134.

Iannetti P, Spalice A, Parisi P (2005) Calcium-channel blocker verapamil administration in prolonged and refractory status epilepticus. Epilepsia 46:967-969.
Immonen RJ, Kharatishvili I, Sierra A, Einula C, Pitkänen A, Gröhn OH (2008) Manganese enhanced MRI detects mossy fiber sprouting rather than neurodegeneration, gliosis or seizure-activity in the epileptic rat hippocampus. Neuroimage 40:1718-1730.

Innis RB, Cunningham VJ, Delforge J, Fujita M, Gjedde A, Gunn RN, Holden J, Houle S, Huang SC, Ichise M, Iida H, Ito H, Kimura Y, Koeppe RA, Knudsen GM, Knuuti J, Lammertsma AA, Laruelle M, Logan J, Maguire RP, et al. (2007) Consensus nomenclature for in vivo imaging of reversibly binding radioligands. J Cereb Blood Flow Metab 27:1533-1539.

Kreisl WC, Liow JS, Kimura N, Seneca N, Zoghbi SS, Morse CL, Herscovitch P, Pike VW, Innis RB (2010) P-glycoprotein function at the blood-brain barrier in humans can be quantified with the substrate radiotracer 11CN-desmethyl-loperamide. J Nucl Med 51:559-566.

Kücker S, Töllner K, Piechotta M, Gernert M (2010) Kindling as a model of temporal lobe epilepsy induces bilateral changes in spontaneous striatal activity. Neurobiol Dis 37:661-672.

Kuntner C, Bankstahl JP, Bankstahl M, Stanek J, Wanek T, Stundner G, Karch R, Brauner R, Meier M, Ding X, Müller M, Löscher W, Langer O (2010) Dose-response assessment of tariquidar and elacridar and regional quantification of P-glycoprotein inhibition at the rat blood-brain barrier using (R)-[11C]verapamil PET. Eur J Nucl Med Mol Imaging 37:942-953.

Kwan P, Brodie MJ (2000) Early identification of refractory epilepsy. N Engl J Med 342:314-319.

la Fougère C, Böning G, Bartmann H, Wängler B, Nowak S, Just T, Wagner E, Winter P, Rominger A, Förster S, Gildehaus FJ, Rosa-Neto P, Minuzzi L, Bartenstein P, Potschka H, Cumming P (2010) Uptake and binding of the serotonin 5-HT1A antagonist [18F]-MPPF in brain of rats: effects of the novel P-glycoprotein inhibitor tariquidar. Neuroimage 49:1406-1415.

Langer O, Bauer M, Hammers A, Karch R, Pataraia E, Koepp MJ, Abrahim A, Luurtsema G, Brunner M, Sunder-Plassmann R, Zimprich F, Joukhadar C, Gentzsch S, Dudczak R, Kletter K, Müller M, Baumgartner C (2007) Pharmacoresistance in epilepsy: a pilot PET study with the p-glycoprotein substrate R-[C-11]verapamil. Epilepsia 48:1774-1784.

Lazarowski A, Sevlever G, Taratuto A, Massaro M, Rabinowicz A (1999) Tuberous sclerosis associated with MDR1 gene expression and drugresistant epilepsy. Pediatr Neurol 21:731-734.

Liow JS, Kreisl W, Zoghbi SS, Lazarova N, Seneca N, Gladding RL, Taku A, Herscovitch P, Pike VW, Innis RB (2009) P-glycoprotein function at the blood-brain barrier imaged using C-11-N-desmethyl-loperamide in monkeys. J Nucl Med 50:108-115.

Löscher W (2007) The pharmacokinetics of antiepileptic drugs in rats: consequences for maintaining effective drug levels during prolonged drug administration in rat models of epilepsy. Epilepsia 48:1245-1258.

Löscher W, Potschka H (2005a) Drug resistance in brain diseases and the role of drug efflux transporters. Nat Rev Neurosci 6:591-602.

Löscher W, Potschka H (2005b) Role of drug efflux transporters in the brain for drug disposition and treatment of brain diseases. Prog Neurobiol 76:22-76.

Luna-Tortós C, Fedrowitz M, Löscher W (2008) Several major antiepileptic drugs are substrates for human P-glycoprotein. Neuropharmacology 55:1364-1375.

Luurtsema G, Molthoff CF, Schuit RC, Windhorst AD, Lammertsma AA, Franssen EJ (2005) Evaluation of (R)-[C-11]verapamil as PET tracer of $\mathrm{P}$-glycoprotein function in the blood-brain barrier: kinetics and metabolism in the rat. Nucl Med Biol 32:87-93.

Muzi M, Mankoff DA, Link JM, Shoner S, Collier AC, Sasongko L, Unadkat JD (2009) Imaging of cyclosporine inhibition of P-glycoprotein activity using 11C-verapamil in the brain: studies of healthy humans. J Nucl Med 50:1267-1275.

Paxinos G, Watson C (2007) The rat brain in stereotaxic coordinates. Sydney: Academic.

Pike VW (2009) PET radiotracers: crossing the blood-brain barrier and surviving metabolism. Trends Pharmacol Sci 30:431-440.

Potschka H, Löscher W (2001) In vivo evidence for P-glycoproteinmediated transport of phenytoin at the blood-brain barrier of rats. Epilepsia 42:1231-1240.

Regesta G, Tanganelli P (1999) Clinical aspects and biological bases of drugresistant epilepsies. Epilepsy Res 34:109-122.

Rizzi M, Caccia S, Guiso G, Richichi C, Gorter JA, Aronica E, Aliprandi M, Bagnati R, Fanelli R, D’Incalci M, Samanin R, Vezzani A (2002) Limbic 
seizures induce P-glycoprotein in rodent brain: functional implications for pharmacoresistance. J Neurosci 22:5833-5839.

Roch C, Leroy C, Nehlig A, Namer IJ (2002) Magnetic resonance imaging in the study of the lithium-pilocarpine model of temporal lobe epilepsy in adult rats. Epilepsia 43:325-335.

Schmitt FC, Merschhemke M, Dehnicke C, Meencke H (2007) Status epilepticus with tonic seizures in nonlesional epilepsy: multidrug transporter inhibitor (verapamil) attenuates malignant treatment course. Epilepsia 48:103-109.

Seneca N, Zoghbi SS, Liow JS, Kreisl W, Herscovitch P, Jenko K, Gladding RL, Taku A, Pike VW, Innis RB (2009) Human brain imaging and radiation dosimetry of C-11-N-desmethyl-loperamide, a PET radiotracer to measure the function of P-glycoprotein. J Nucl Med 50:807-813.

Sharom FJ (1997) The P-glycoprotein efflux pump: how does it transport drugs? J Membr Biol 160:161-175.

Sisodiya SM, Thom M (2003) Widespread upregulation of drug-resistance proteins in fatal human status epilepticus. Epilepsia 44:261-264.

Sisodiya SM, Lin WR, Harding BN, Squier MV, Thom M (2002) Drug resistance in epilepsy: expression of drug resistance proteins in common causes of refractory epilepsy. Brain 125:22-31.

Stein WD, Cardarelli C, Pastan I, Gottesman MM (1994) Kinetic evidence suggesting that the multidrug transporter differentially handles influx and efflux of its substrates. Mol Pharmacol 45:763-772.

Syvänen S, Lindhe O, Palner M, Kornum BR, Rahman O, Långström B, Knudsen GM, Hammarlund-Udenaes M (2009) Species differences in blood-brain barrier transport of three positron emission tomography ra- dioligands with emphasis on P-glycoprotein transport. Drug Metab Dispos 37:635-643.

Tishler DM, Weinberg KI, Hinton DR, Barbaro N, Annett GM, Raffel C (1995) Mdr1 gene-expression in brain of patients with medically intractable epilepsy. Epilepsia 36:1-6.

van Vliet EA, da Costa Araújo S, Redeker S, van Schaik R, Aronica E, Gorter JA (2007a) Blood-brain barrier leakage may lead to progression of temporal lobe epilepsy. Brain 130:521-534.

van Vliet EA, van Schaik R, Edelbroek PM, Voskuyl RA, Redeker S, Aronica E, Wadman WJ, Gorter JA (2007b) Region specific overexpression of P-glycoprotein at the blood-brain barrier affects brain uptake of phenytoin in epileptic rats. J Pharmacol Exp Ther 332:141-147.

Volk HA, Potschka H, Löscher W (2004) Increased expression of the multidrug transporter P-glycoprotein in limbic brain regions after amygdalakindled seizures in rats. Epilepsy Res 58:67-79.

Wagner CC, Bauer M, Karch R, Feurstein T, Kopp S, Chiba P, Kletter K, Löscher W, Müller M, Zeitlinger M, Langer O (2009) A pilot study to assess the efficacy of tariquidar to inhibit P-glycoprotein at the human blood-brain barrier with (R)-C-11-verapamil and PET. J Nucl Med 50:1954-1961.

Wahnschaffe U, Löscher W (1992) Lack of changes in seizure susceptibility during the estrous-cycle in kindled rats. Epilepsy Res 13:199-204.

Zoghbi SS, Liow JS, Yasuno F, Hong J, Tuan E, Lazarova N, Gladding RL, Pike VW, Innis RB (2008) C-11-loperamide and its N-desmethyl radiometabolite are avid substrates for brain permeability-glycoprotein efflux. J Nucl Med 49:649-656. 